\title{
Characteristics analysis of water film in transpiring wall reactor
}

\author{
Donghai $\mathrm{Xu}^{*}$, Chuanbao Huang, Shuzhong Wang*, Yang Guo \\ Key Laboratory of Thermo-Fluid Science \& Engineering, Ministry of Education, School of Energy \\ and Power Engineering, Xi'an Jiaotong University, Xi'an, Shaanxi Province 710049, China
}

\begin{abstract}
Transpiring wall reactor can significantly overcome reactor material corrosion and salt deposition problems by forming a protective water film on its inner porous wall. In this work, a simplified physical model of transpiring wall reactor was built to analyze the process of heat and mass transfer between the water film and bulk fluid. Furthermore, two quantitative correlations between the thickness of water film and main operating parameters were proposed for the first time using theoretical analysis and mathematical deduction method. For a given transpiring wall reactor, appropriately increasing the flow rate and inlet temperature of transpiration water is conducive to maintain the growth of the water film thickness. A positive linear relation exists between the mass flow rate of transpiration water and the thickness of water film under constant feedstock parameters conditions. These quantitative correlations together with some meaningful conclusions help to guide the optimization of operating parameters as well as the design of transpiring wall reactor.
\end{abstract}

Keywords: Transpiring wall reactor, water film, transpiration water, porous wall, heat transfer

\footnotetext{
* Corresponding author. Tel.: +86-29-82665749; fax: +86-29-8266-8703.

E-mail address: haidongxu93346423@aliyun.com (D.H. Xu), szwang@aliyun.com (S.Z. Wang).
} 


\section{Introduction}

Water above its critical point $\left(\mathrm{T}=374^{\circ} \mathrm{C}, \mathrm{P}=22.1 \mathrm{MPa}\right)$ exhibits certain unique properties, such as non-polar character, high diffusivity, excellent dissolution and transportation features[1]. Supercritical water oxidation (SCWO) utilizes these excellent properties to achieve rapid and complete degradation of organic waste in the presence of excess oxygen. SCWO possesses some prominent advantages such as very short reaction time, easier salts separation, achieving heat self-sufficiency and absence of secondary pollutants[2]. It is feasible for the harmless treatment of high toxicity, high concentration and refractory organic waste. However, its core equipment (i.e., reactor), easily suffers with serious corrosion due to the harsh working conditions and plugging problems caused by salt deposition[3]. Innovative reactor design is an effective means to solve these two problems, wherein transpiring wall reactor (TWR) attaches much attention. The reactor consists of outer pressure-bearing wall and inner porous non-load-bearing transpiring wall. A protective water film, covering the inner surface of the porous wall, can effectively isolates corrosion substances and inorganic salts, so reducing the material corrosion rate and the risk of salt deposition. Numerous experiments regarding organic matter removal, material corrosion and salt deposition have been conducted in transpiring wall reactor SCWO plants[2, 4-7]. The experimental results indicate that transpiring wall reactor plays an effective role in resistance to corrosion and salt deposition[8-11].

However, there are no in-depth studies concerning the impacts of operating parameters on the water film characteristics (such as the thickness, uniformity and continuity), resulting in some inconsistent, even contradictory conclusions on the performance of TWR. For instance, Rice et al.[8], Crooker et al.[9] and $\mathrm{Xu}$ et al.[10] reported that no obvious corrosion and salt deposition 
phenomena were observed in SCWO of actual wastewater or simulated salt-containing wastewater. Nevertheless, Fauvel et al.[12, 13] and Gonget al.[6, 14] discoveried that porous transpiring wall was seriously corroded and plugged by inorganic salts after a certain period of time. Water film characteristics seem to be a key factor to exert the advantages of transpiring wall reactor, while operating parameters have decisvie impacts on it. It has been proven that the higher transpiration intensity helps to form a better water film, but excess cool transpiration water easily results in the fluctuation of temperature and the recduction of organics removal efficiency near the water film[1]. Therefore, it is necessary to seek appropriate correlations between the water film characteristics and operating parameters to guide reactor design and optimization of operating parameters.

Till now, some researchers have investigated heat transfer and dynamics of supercritical fluid in the porous medium. Reda et al.[15] studied the mixed convection phenomenon in vertical porous medium following Darcy law. Fauvel et al.[13] discussed the pressure drop of supercritical fluid in the porous wall governed by Darcy law as well. Jiang et al.[16] obtained the correlations of describing pressure drop and convective heat transfer coefficient, considering multiple factors such as non-Darcy effect, inertia term, thermal dispersion, variable porosity, variable properties, buoyancy and particle diameter. These factors strongly influence heat transfer and flow characteristics of the fluid in porous medium. Chen et al.[1] experimentally observed that the fluid displayed anisotropy across a porous wall by a visual glass apparatus imitating SCWO conditions. Bermejo et al.[17] investigated the effects of the porous type, pore size and reaction temperature on the protective water film in a transpiring wall reactor at constant operating conditions. On the whole, existing literature concerning the porous medium or transpiring wall mainly concentrates on the temperature profiles, pressure drop and heat transfer coefficient. The studies did not 
consider the process of heat mass between the water film and bulk fluid, which was helpful to guide reactor design and the optimization of operating parameters.

In respect to transpiring wall reactor, the influence of operating conditions (i.e., transpiration intensity and the temperature of transpiration water ) on the reactor performance including the temperature profiles, salt deposition, product distributions and organic matter conversion rate have been studied extensively[4-6, 17-20]. For instance, Wellig et al.[18] experimentally studied the effects of operating characteristics of transpiring wall SCWO reactor on the temperature profiles near the transpiring and pressure-bearing wall. They pointed out that the natural convection effects were not negligible in the reactor, but no in-depth studies were carried out to investigate the convection effect and more operating conditions (i.e., the temperature and flow rate of bulk fluid in the reactor ) on the formation of water film. Furthermore, some process parameters were optimized based on the temperature profiles and gas-liquid products in a transpiring wall reactor SCWO pilot plant by Zhang et al.[20]. Some calculated models of TWRs are also proposed to simulate and improve the performance of the reactor[1, 12, 21-24]. Bermejo et al.[22] established a simplified model of a TWR and studied the effects of transpiring water temperature, flow rate and composition of the oxidant on the reactor performance such as the temperature and composition contours, flow path lines and effluent compositions using the commercial software Fluent 6.3. Zhang et al.[24] also presented a numerical model of TWR for SCWO and discussed the effect of the transpiration intensity and the transpiring water temperature on the temperature profile and TOC removal rate in the reactor. Moreover, the temperature of water film was optimized by controlling the inner surface temperature of the porous tube less than $374^{\circ} \mathrm{C}$. However, these studies did not consider the effects of other operating conditions such as the 
feedstock parameters (i.e., the temperature and concentration of organics) on the formation of the water film, lacking the optimization means of TWR for a better water film in resistance to corrosion and salt deposition. The flow and heat transfer characteristics between its protective water film and bulk fluid need to be deeply explored due to their crucial roles in the formation of the water film. To the best of our knowledge, there are no related investigations concerning the characteristics of the water film in a TWR presently. No concrete and quantitative theory is applicable to the optimization of water film characteristics such as the thickness. Nonetheless, these characteristics are decisive for the ultimate realization of reactor's functions minimizing corrosion and salt deposition. A novel TWR and its SCWO system had been developed in previous reports[10, 25]. In this paper, a simplified model of TWR was built to theoretically analyze the heat and mass transfer processes between its water film and bulk flow, based on mass, momentum and energy governing equations. Two quantitative relations are firstly proposed to describe the relationship between the water film characteristics and operating parameters in TWR, which were available to guide the reactor design and optimize operating parameters. As an extension of our previous research, this is firstly documented in the literature.

\section{Nomenclature}

$\begin{array}{ll}A & \text { Surface area of porous wall, } \mathrm{m}^{2} \\ c_{\mathrm{p}} & \text { Specific heat of transpiration water, } \mathrm{J} /(\mathrm{kg} \cdot \mathrm{K}) \\ D & \text { Generalized diffusion coefficient, } \mathrm{m}^{3} / \mathrm{s} \\ d & \text { Porous wall diameter, } \mathrm{m} \\ F_{\mathrm{c}} & \text { Mass flow rate of transpiration water, } \mathrm{kg} / \mathrm{s} \\ g & \text { Gravity acceleration, } \mathrm{m} / \mathrm{s}^{2} \\ G a & \text { Galileo number }\end{array}$




\begin{tabular}{|c|c|}
\hline$l$ & Porous wall length, m \\
\hline$p$ & Pressure, $\mathrm{Pa}$ \\
\hline $\operatorname{Pr}$ & Prandtl number \\
\hline$q_{\mathrm{m}}$ & Mass flow rate through one transpiration water section, $\mathrm{kg} / \mathrm{s}$ \\
\hline$\delta Q(z)$ & Heat transferred across the differential surface area, w \\
\hline$t_{\mathrm{c}}(\mathrm{z})$ & Average temperature of the water film, $\mathrm{K}$ \\
\hline$t_{\mathrm{i}}(z)$ & Outer surface temperature of the water film, $\mathrm{K}$ \\
\hline$t_{\mathrm{w}}(z)$ & Porous wall temperature, $\mathrm{K}$ \\
\hline$t_{0}(\mathrm{z})$ & Inlet temperature of transpiration water, $\mathrm{K}$ \\
\hline$T_{0}$ & Inlet temperature of bulk fluid, $\mathrm{K}$ \\
\hline$u_{z}$ & Transpiration water velocity at the $\mathrm{z}$-direction, $\mathrm{m} / \mathrm{s}$ \\
\hline$u_{r}$ & Transpiration water velocity at the $\mathrm{r}$-direction, $\mathrm{m} / \mathrm{s}$ \\
\hline$u_{\theta}$ & Transpiration water velocity at the $\theta$-direction, $\mathrm{m} / \mathrm{s}$ \\
\hline$U$ & Bulk fluid velocity, $\mathrm{m} / \mathrm{s}$ \\
\hline$r, \theta, z$ & Coordinates, $\mathrm{m}$ \\
\hline
\end{tabular}

Greek symbols

$\begin{array}{ll}\delta & \text { Water film thickness, } \mathrm{m} \\ \lambda & \text { Thermal conductivity, } \mathrm{w} /(\mathrm{m} \cdot \mathrm{K}) \\ \mu & \text { Dynamic viscosity, } \mathrm{Pa} \cdot \mathrm{s} \\ \rho & \text { Fluid density, } \mathrm{kg} / \mathrm{m}^{3}\end{array}$

Subscripts

$0 \quad$ Cool transpiration water inlet

c Cool transpiration water

bulk Bulk fluid

i Interface between water film and bulk fluid

m Mass flow rate

W Porous wall

$1 \quad$ Mass loss 


\section{Physical model and theoretical analysis}

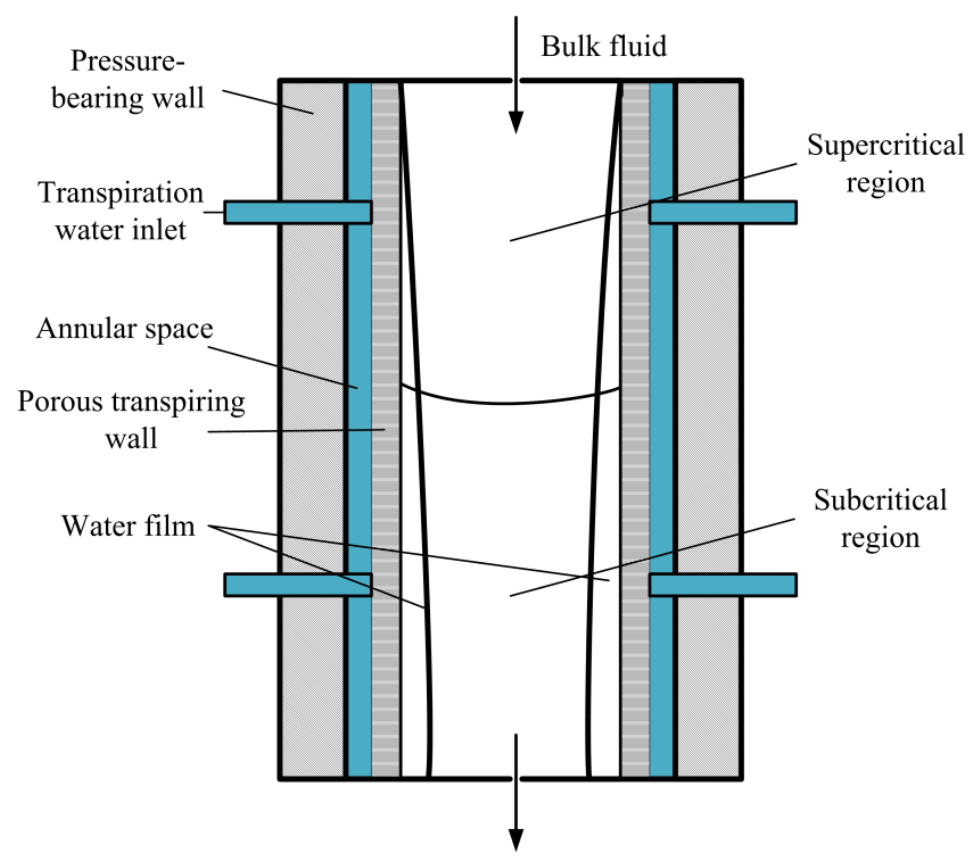

Fig. 1 Typical structure and flow pattern in transpiring wall reactor

As shown in Fig.1, transpiration water is pumped into the annular space between the pressure-bearing wall and the non-load-bearing porous transpiring wall, and gradually pervades the inner side of the porous wall with the driving effect of differential pressure. Bulk fluid (reaction fluid) is introduced from the reactor top, and restricted in the central zone where oxidation reactions take place and form a supercritical region there[5]. The mixture of reaction products and cool transpiration water flows downward by gravity, forming a subcritical region at the bottom of reactor. Ideally, a uniform and continuous water film covers the whole porous wall exposed to the reaction fluid and products, cutting off wall contact with corrosive species and inorganic salts. The solid salts precipitated in the upper supercritical region can re-dissolve in the subcritical region, which are eventnually discharged from the bottom outlet of the reactor[17].

The average temperature of the water film is an important indicator to evaluate its quality. Controlling the temperature below the water's critical point not only prevents two reactor walls 
over temperature limitation, but also further sweeps, dissolves and dilutes inorganic salts and other corrosive substances close to the transpiring wall. Notably, the upper water film near oxidation reaction region is easy to reach a supercritical temperature as a large amount of heat is released in a very short time. Hence, it's crucial to maintain a subcritical water film temperature in the reaction zone.

Assuming that the water film is continuous and isotropic along the circumferential direction of the reactor with the same characteristic parameters at the same height. Thus, the three-dimensional TWR can be simplified into a 2D cylindrical coordinate model taking into account that the typical reactor has a symmetrical structure (see Fig.1). As shown in Fig.2, the model describes the downward flow of transpiration water and bulk fluid in a vertical cylinder with a uniform and isotropic porous medium. The bulk fluid enters the reactor with a uniform velocity $U$ and a constant temperature $T_{0}$, while the transpiration water is introduced from both sides of the reactor with the inlet temperature of $t_{0}$. The flow state of transpiration water in the porous medium is considered to be steady, two dimensional and governed by Darcy law due to the small wall thickness. Therefore, transpiration water is idealized as flowing downward along the inner surface of the porous wall with the help of gravity and bulk flow impulse. The water film's thickness $\delta$ continuously increases to a stable value while its axial velocity $u_{z}$ increases to $99 \%$ of the bulk fluid velocity $U$. Solid phase and liquid phase in porous medium keep heat balance, and the temperature $t_{0}$ may keep a constant or variable value depending on operating conditions. Considering variable properties and gravity acceleration, the steady state, two dimensional governing equations for the flow fluid in the TWR can be written in the following forms

$$
\frac{1}{r} \frac{\partial\left(r u_{r}\right)}{\partial r}+\frac{\partial u_{z}}{\partial z}=0
$$




$$
\begin{gathered}
u_{r} \frac{\partial u_{z}}{\partial r}+u_{z} \frac{\partial u_{z}}{\partial z}=g-\frac{1}{\rho} \frac{\partial p}{\partial z}+v\left(\frac{\partial^{2} u_{z}}{\partial r^{2}}+\frac{1}{r} \frac{\partial u_{z}}{\partial r}+\frac{\partial^{2} u_{z}}{\partial z^{2}}\right) \\
u_{r} \frac{\partial t}{\partial r}+u_{z} \frac{\partial t}{\partial z}=\alpha\left[\frac{1}{r} \frac{\partial}{\partial r}\left(r \frac{\partial t}{\partial r}\right)+\frac{\partial^{2} t}{\partial z^{2}}\right]
\end{gathered}
$$

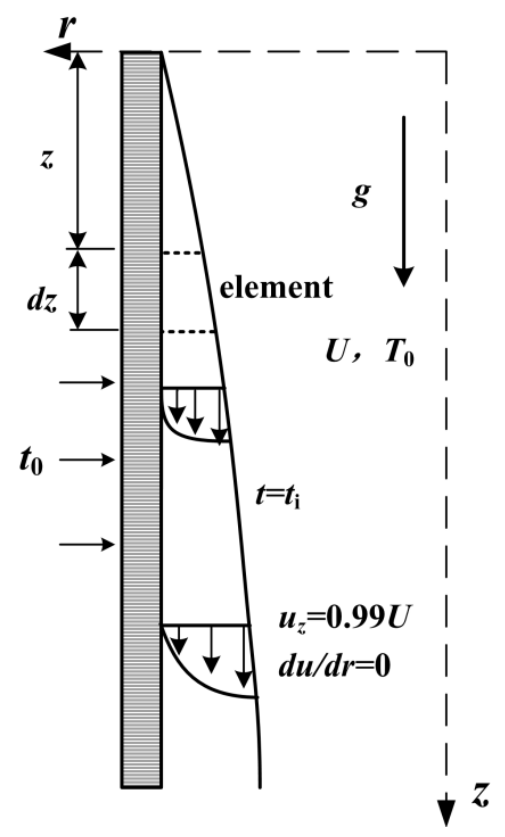

Fig. 2 Physical model of flow in transpiring wall reactor.

In order to obtain analytical solutions, these governing equations require to be further simplified.

The second order derivative of the velocity and the temperature at the axial direction corresponding to the momentum equation (2) and energy equation (3) respectively can be ignored according to the analysis of order of magnitude method[26]. Thus, the simplified governing equations are as followss

$$
\begin{gathered}
\frac{1}{r} \frac{\partial\left(r u_{r}\right)}{\partial r}+\frac{\partial u_{z}}{\partial z}=0 \\
\rho\left(u_{r} \frac{\partial u_{z}}{\partial r}+u_{z} \frac{\partial u_{z}}{\partial z}\right)=\rho g-\frac{d p}{\partial z}+\mu\left(\frac{\partial^{2} u_{z}}{\partial r^{2}}+\frac{1}{r} \frac{\partial u_{z}}{\partial r}\right) \\
u_{r} \frac{\partial t}{\partial r}+u_{z} \frac{\partial t}{\partial z}=\alpha\left[\frac{1}{r} \frac{\partial}{\partial r}\left(r \frac{\partial t}{\partial r}\right)\right]
\end{gathered}
$$


In views of the extreme complex process of heat and mass transfer between water film and bulk fluid, some necessary assumptions and explanations are made for the water film as follows

1) The water film is laminar flow, which can cover the entire porous wall.

2) Inertia term in the momentum equation is negligible due to low velocity and Reynolds number (laminar flow) in the water film.

3) Given that the heat transfer mode in the water film is only heat conduction, of which the temperature distribution can be considered to be linear.

Assumptions for both the water film and bulk fluid in TWR are listed as follows

4) The interface between water film and bulk fluid is smooth without viscous stress.

5) Heat transfer on the interface of water film and bulk fluid can be regarded as convection heat transfer in a single phase without apparent phase transition process at the supercritical pressure condition.

6) Mass exchange between transpiration water and bulk flow keeps a dynamic balance because it is difficult to quantitatively predict or calculate the variation during the complex diffusion and mixing process.

7) Hypothesize that the pressure is constant along the radial direction in the reactor.

The inertia term in the momentum equation (5) can be ignored according to the previous assumption 2). Moreover, the pressure function $p$ can be expressed as $p=p(z)$ in terms of the assumption 7). Thus, the pressure gradient term introduced by the momentum equation (5) can be calculated by

$$
\frac{\partial p}{\partial z}=\frac{d p}{d z}=\rho_{\mathrm{i}} g
$$

where $\rho_{\mathrm{i}}$ represents the density of the water film outside surface at the position of $r=R-\delta$, which 
can be obtained by querying the thermo-physical property database in terms of the corresponding temperature $t_{\mathrm{i}}$.

The right term of energy equation (6) can be negligible in accordance with the assumption 3).

Thus, Eqs. (4-6) are further simplified in terms of the above assumptions:

$$
\begin{gathered}
\frac{1}{r} \frac{\partial\left(r u_{r}\right)}{\partial r}+\frac{\partial u_{z}}{\partial z}=0 \\
\left(\rho_{\mathrm{c}}-\rho_{\mathrm{i}}\right) g+\mu\left(\frac{\partial^{2} u_{z}}{\partial r^{2}}+\frac{1}{r} \frac{\partial u_{z}}{\partial r}\right)=0 \\
{\left[\frac{1}{r} \frac{\partial}{\partial r}\left(r \frac{\partial t}{\partial r}\right)\right]=0}
\end{gathered}
$$

The corresponding boundary conditions in these equations are

when $r=d / 2$ :

$$
u_{z}=0, t=t_{\mathrm{w}}
$$

when $\mathrm{r}=d / 2-\delta$ :

$$
\frac{\partial u_{z}}{\partial r}=0, t=t_{\mathrm{i}}
$$

where $\rho_{\mathrm{c}}$ represents the average density of the water film obtained by querying the thermo-physical property database depending on the corresponding temperature $t_{\mathrm{c}}$. And the qualitative temperature of water film, $t_{\mathrm{c}}$, is expressed as

$$
t_{\mathrm{c}}=\frac{t_{\mathrm{i}}+t_{\mathrm{w}}}{2}
$$

where $\mu$ is the dynamics viscosity, the temperature of inner wall, $t_{\mathrm{w}}$, is measured by specially arranged thermocouples, and $\delta$ is the thickness of water film. The interface temperature $t_{\mathrm{i}}$, which are available by further experiment and numerical simulation data. 
By solving Eqs. (8-12), the velocity and the temperature relations in the water film are as followss

$$
\begin{gathered}
u_{z}=-\frac{\left(\rho_{\mathrm{c}}-\rho_{\mathrm{i}}\right) g}{4 \mu}\left[r^{2}+2\left(\frac{d}{2}-\delta\right)^{2} \ln \frac{d}{2 r}-\left(\frac{d}{2}\right)^{2}\right] \\
t=t_{\mathrm{w}}+\left(t_{\mathrm{i}}-t_{\mathrm{w}}\right) \frac{\ln (2 r / d)}{\ln (1-2 \delta / d)}
\end{gathered}
$$

Further, the mass balance of the $d z$ element is analyzed to determine the thickness of water film $\delta$.

As indicated in Fig. 3, the mass flow rate through the cross section, $q_{m}$, can be calculated by

$$
q_{\mathrm{m}}=\int_{(d-2 \delta) / 2}^{d / 2} \rho_{\mathrm{c}} u_{z}\left[2 \pi r d r=\frac{\pi \rho_{\mathrm{c}}^{2} g}{2 \mu}\left\{\left(\frac{d}{2}-\delta\right)^{4}\left(\frac{3}{4}+\ln \frac{d / 2}{d / 2-\delta}\right)-\left[\frac{d}{2}\left(\frac{d}{2}-\delta\right)\right]^{2}+\frac{d^{4}}{64}\right\}\right.
$$

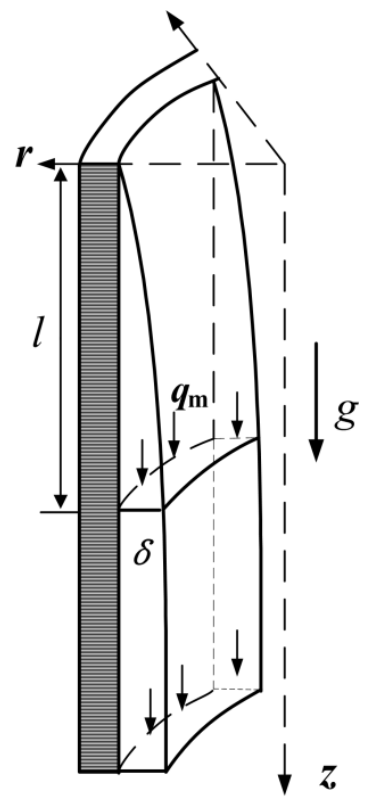

Fig. 3 Schematic diagram of water film flowing through the cross section

Considering that the film thickness $\delta$ is far less than the porous wall diameter $d$ in the scale, thus, the logarithmic term $\ln [d /(d-2 \delta)]$ is approximately zero in Eq. (16), and the expression for $q_{\mathrm{m}}$ is simplified as

$$
q_{\mathrm{m}}=\frac{\pi \rho_{\mathrm{c}}^{2} g}{2 \mu}\left\{\frac{3}{4}\left(\frac{d}{2}-\delta\right)^{4}-\left[\frac{d}{2}\left(\frac{d}{2}-\delta\right)\right]^{2}+\frac{d^{4}}{64}\right\}
$$


The increment of the mass flow rate in the $d z$ element, i.e., the derivative of $q_{m}$, is calculated by

$$
d q_{\mathrm{m}}=-\frac{\pi \rho_{\mathrm{c}}^{2} g}{2 \mu}\left\{\left[3\left(\frac{d}{2}-\delta\right)^{3}-d\left(\frac{d}{2}-\delta\right)\right] d \delta\right\}
$$

Note that the heat transfer balance in the $d z$ element is analyzed without considering radiation due to the lower reaction temperature. As illustrated in Fig. 4, heat conduction through the water film thickness $\delta$ is equal to the heat absorption increment $\left(d q_{m}\right)$ of the water film in the $d z$ element. The heat transfer $\delta Q(z)$ in the element can be obtained by

$$
\delta Q(z)=\lambda \frac{t_{\mathrm{i}}(z)-t_{\mathrm{w}}(z)}{\delta} \cdot 2 \pi\left(\frac{d}{2}-\delta\right) d z=c_{\mathrm{p}} \cdot d q_{\mathrm{m}} \cdot\left[t_{\mathrm{tw}}(z)-t_{0}(z)\right]
$$

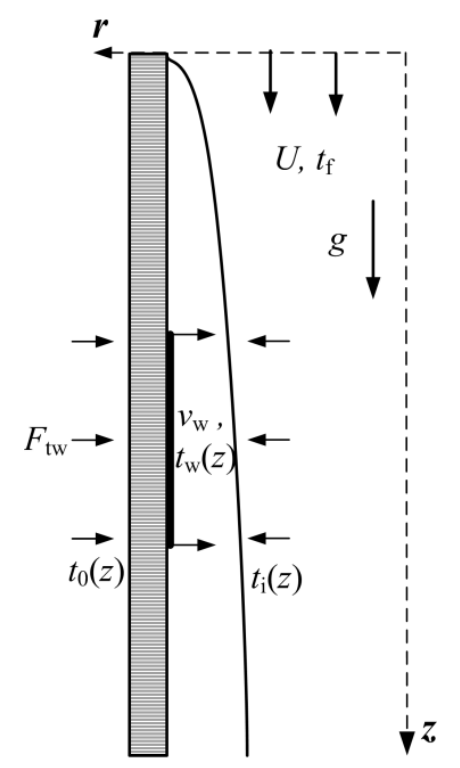

Fig. 4 Heat transfer balance between water film and bulk fluid.

Eq. (19) continues to be integrated as

$$
\delta^{2}\left[\left(\delta-\frac{2}{3} d\right)^{2}+\frac{1}{18} d^{2}-2 d\right]=-\frac{4 \lambda \mu\left(t_{\mathrm{i}}(z)-t_{\mathrm{w}}(z)\right)}{c_{\mathrm{p}} \rho_{\mathrm{c}}^{2} g\left(t_{\mathrm{tw}}(z)-t_{0}(z)\right)} z
$$

where $\lambda$ represents the thermal conductivity of water film, and $c_{\mathrm{p}}$ is specific heat capacity at constant pressure. 
The dimensionless parameters $\mathrm{Ga}, \mathrm{Pr}$ are introduced into Eq. (20) to further simplify the expression as

$$
\delta^{2}\left[\left(\delta-\frac{2}{3} d\right)^{2}+\frac{1}{18} d^{2}-2 d\right]=-\frac{C_{1} L^{3}\left(t_{\mathrm{i}}(z)-t_{\mathrm{w}}(z)\right)}{\operatorname{Pr} \cdot G a \cdot\left(t_{\mathrm{tw}}(z)-t_{0}(z)\right)} z
$$

where

$$
G a=\frac{g L^{3} \rho_{\mathrm{c}}^{2}}{\mu^{2}}, \operatorname{Pr}=\frac{\mu c_{\mathrm{p}}}{\lambda}
$$

$C_{1}$ is the thickness corrected coefficient of water film obtained by experimental measurement data, and $L$ is the length of TWR.

Furthermore, the correlation of the transpiration water flow rate can be expressed as

$$
F_{\mathrm{c}}=C_{2} \frac{\pi \rho_{\mathrm{c}}^{2} g}{\mu}\left\{(d-2 \delta(L))^{4}\left(\frac{3}{4}+\ln \frac{d}{d-2 \delta(L)}\right)-[d(d-2 \delta(L))]^{2}+\frac{d^{4}}{4}\right\}
$$

\section{Results and Discussions}

It's difficult to calculate quantitatively due to some unknown parameters in Eq. (21) such as the thickness corrected coefficient $C_{1}$, and the interface temperature $t_{\mathrm{i}}$, which are available by further experiment and numerical simulation data. Thus, the general trends of water film thickness at different positions are displayed as shown in Fig.5. For a given TWR, the thickness of water film continuously increases along the reactor length, and the high growth rate at the initial stage of the reactor gradually decreases with the increase in the distance from the reactor inlet. The thickness of water film tends to be stable in subcritical region at the bottom of the reactor, which has a better protection effect on the porous wall. On the other hand, the thickness of 
water film at the same position decreases with the increase of Prandtl number, which shows that the larger Prandtl number is not conducive to the formation of water film. However, the value of Prandtl number exhibits non-monotonic trends under the operating conditions (such as the pressure and temperature). As shown in Fig. 6, the Prandt1 number has an approximate positive correlation with the pressure within the range of operating pressure (23-32MPa). But it first increases and then reduces following the increase of the temperature, which exists an inflection point in the vicinity of the critical point. Therefore, it's necessary to further analyze the influence of operating parameters on the formation and thickness of water film.

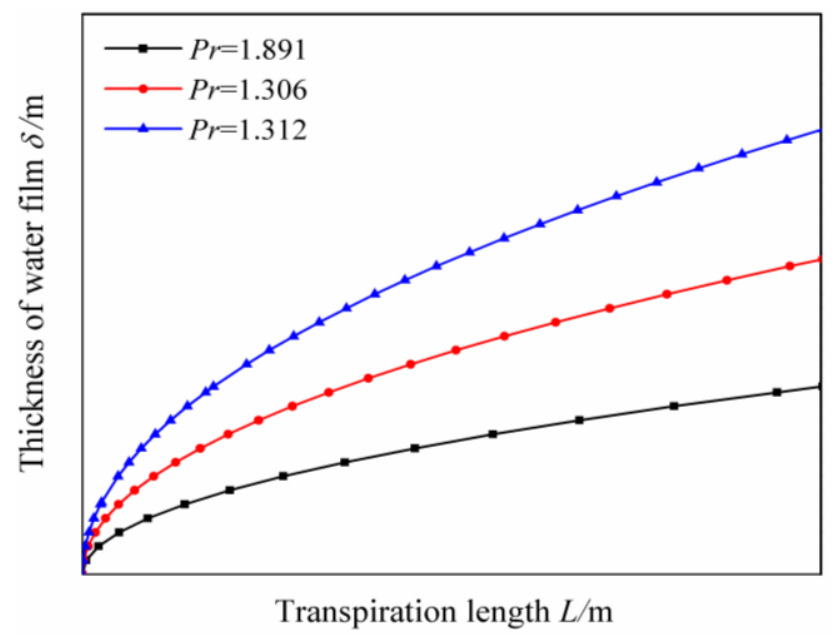

Fig.5 Trends of the water film thickness with TWR transpiration length 


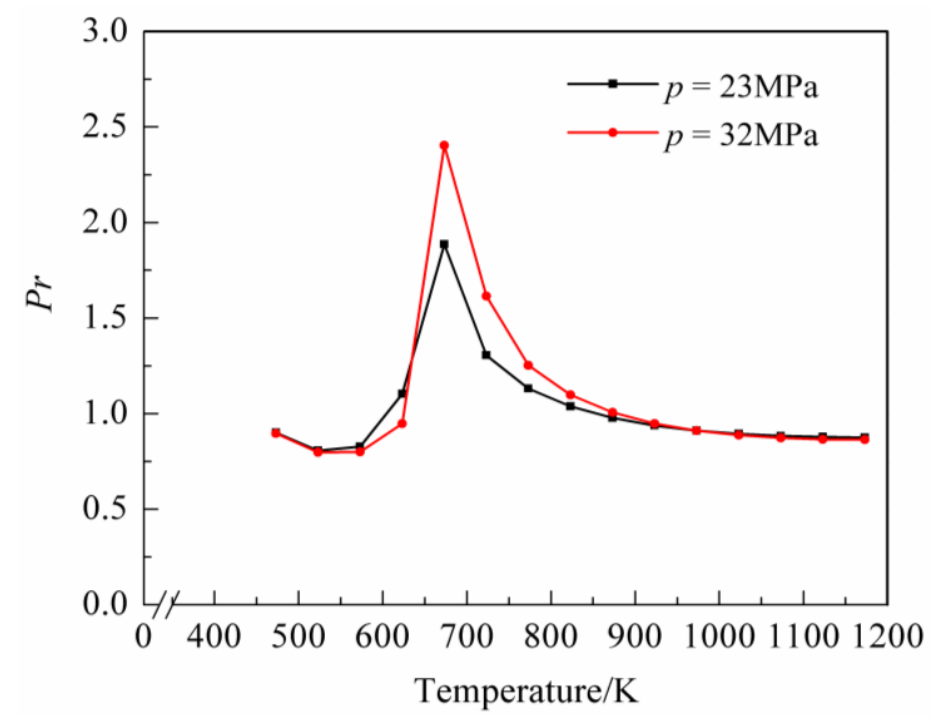

Fig.6 Prandtl number of the water film at different pressure and temperature

Certainly, the correlation contains some unknown parameters obtained by experiments and/or numerical simulation. It is believed that Eqs. (21-23) may provide relatively accurate information for the thickness of water film. However, it cannot directly qualitatively reflect the trends of reactor structure size and operating parameters, which is still too complex for application in the optimization of operating parameters and reactor design. Thus, these mathematical correlations needs to further simplified. Herein, two more assumptions are given before discussions. One is to neglect the influence of gravity acceleration on the distribution of the water film. Another is that water film uniformly distributes on the inner surface of the porous wall.

Transpiration water with mass flow rate of $F_{\mathrm{c}}$ forms an initial water film with a certain thickness.

The initial thickness $\delta_{0}$ can be calculated by

$$
\delta_{0}=\frac{F_{\mathrm{c}}}{A \rho_{\mathrm{c}}}
$$

The loss of the mass flow rate caused by diffusion effect is approximately calculated by 


$$
F_{1}=\frac{2 A D\left(\rho_{\mathrm{c}}-\rho_{\text {bulk }}\right)}{d}
$$

where $F_{1}$ represents the flow rate loss, which is determined by the density difference between water film and bulk fluid. The water film area $A$ is calculated by using $A=2 \pi R l$. The diffusion coefficient, $D$, can be gained by experiments. $\rho_{\text {bulk }}$ and $\rho_{\mathrm{c}}$ represent the densities of bulk fluid and transpiration water, respectively. Assume that transpiration water uniformly covers on the inner surface of the porous wall. The actual thickness of the water film with the consideration of the loss of mass flow rate can be obtained by

$$
\delta=\delta_{0}-\delta_{\text {loss }}=\frac{F_{\mathrm{c}}}{A \rho_{\mathrm{c}}}-\frac{2 D}{d} \cdot\left(1-\frac{\rho_{\text {bulk }}}{\rho_{\mathrm{c}}}\right)
$$

The influence of operating parameters on the thickness of water film can be analyzed according to Eq.(26). As shown in Fig.7, transpiration intensity directly affects the formation and thickness of water film, which has a positive linear relation with the thickness for a given TWR. However, increasing the transpiration intensity may cause water film flow state transition from laminar to turbulent flow. The transpiration water sprays into the reactor flowing through the porous aperture with a high speed, which is unfavorable to form a continuous and stable water film. Moreover, excessive transpiration water inevitably reduces the removal efficiency of organics and leads to undesirable fluctuation of temperature and pressure in the reactor. Thus, the transpiration intensity must be controlled in a reasonable range to ensure laminar flow in water film. The range requires further experimental tests to determine. 


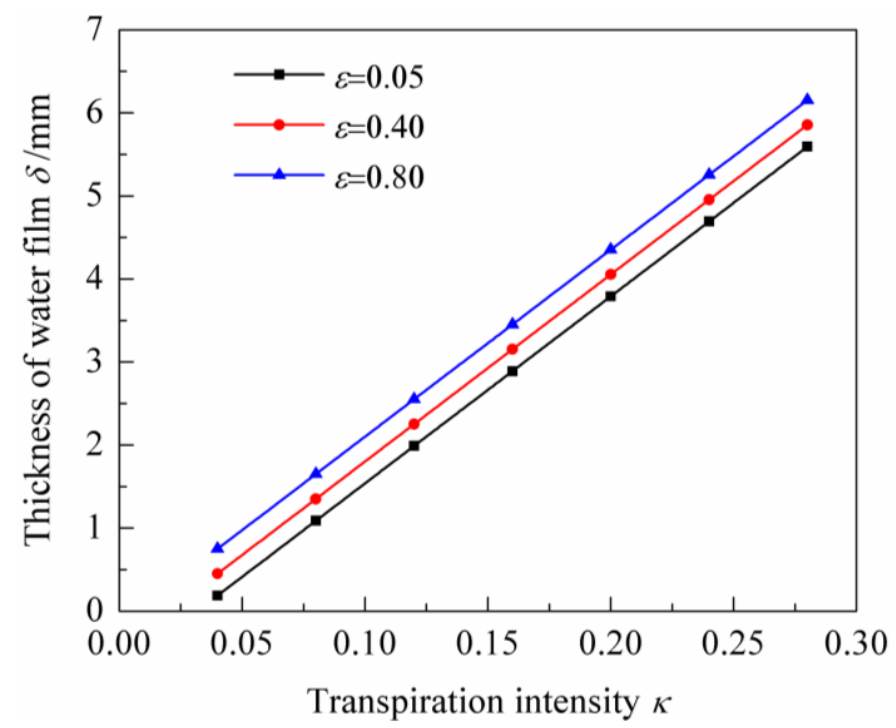

Fig.7 Thickness of water film vs. the transpiration intensity $p=23 \mathrm{MPa}, F_{\text {bulk }}=1.1 \mathrm{~kg} \cdot \mathrm{h}^{-1}, T_{\mathrm{tw}}=573 \mathrm{~K}, D=1 \times 10^{-6} \mathrm{~m}^{2} \cdot \mathrm{s}^{-1}$

The convection and diffusion play an important role in the formation of water film in SCWO reactor[18]. Thus, a new evaluating indicator, density difference coefficient $\varepsilon$, is introduced into Eq.(26) to describe the effects of the convection and diffusion on the film thickness. The density difference coefficient is the density ratio of the bulk fluid and water film, and the expression is as followss

$$
\varepsilon=\frac{\rho_{\text {bulk }}}{\rho_{\mathrm{c}}}
$$

where $\varepsilon$ is less than 1 due to $T_{\text {bulk }}>T_{\mathrm{c}}$, the smaller $\varepsilon$ means greater density difference and more loss of transpiration water caused by the convection diffusion and mass transfer. As shown in Fig.7, the thickness of water film increases with the increase in the value of $\varepsilon$, which is helpful to the formation of water film.

The density is determined by the temperature under the constant pressure condition in TWR. Increasing the temperature of bulk fluid will decrease its density, and corresponding density difference coefficient $\varepsilon$ will also reduces, which results in the reduction of water film thickness 
(see Fig.7). Thus, the variations in operational parameters, such as increasing the concentration, flow rate and preheating temperature of organic feedstock, are unfavorable for the formation of water film. Correspondingly, reducing the temperature of water film also decreases the density difference coefficient $\varepsilon$, causing reduction in the thickness of water film. As a result, increasing the inlet temperature of water film and choosing lower transpiration intensity can weaken the convection diffusion effects between the bulk fluid and water film as well as decrease the density of transpiration water $\left(\rho_{c}\right)$ in Eq. (26), so obtaining a better protective water film. However, the high inlet temperature of transpiration water causes thermal damage of the porous wall and weakens the dissolution and dilution effects on inorganic salts. Moreover, feedstock parameters (especially the organic concentration and flow rate) need to match the transpiration intensity. Otherwise, the higher flow rate or concentration of organic matter in the feedstock may result in a sharp increment in temperature of bulk fluid. The elevated temperature of bulk flow increases the diffusion loss of transpiration water caused by higher density difference between water film and bulk fluid. As a consequence, the water film thickness drastically reduces or even disappears after certain period of running time. Essentially, this correlation (i.e., Eq. (25)) can be used to qualitatively explain some phenomena in typical TWR.

The Eq.(26) is convenient for analyzing the formation mechanisms and influencing factors of water film, and further optimizing initial operating parameters for forming a continuous and stable protective water film. However, the simplified correlation ignores some important influence factors such as the gravity and static pressure, so the correlation will be restricted in the practical application.

Notably, heat transfer between water film and bulk fluid in SCWO process is regarded as a single 
phase convection process. Thus, the water film thickness can be calculated by the following form

$$
\delta=\frac{\lambda(z)}{h(z)}
$$

where local heat transfer coefficient, $h(z)$, can be measured and calculated by relevant experiments in transpiring wall reactor. $\lambda(z)$ represents the local thermal conductivity of the water film, which can be obtained from the database of thermodynamic properties corresponding to temperature $t(z)$. Therefore, the aforementioned two correlations (Eq. (21) and Eq. (23)) verified by experimental data in following work can be used to estimate the thickness of the water film for a given transpiring wall reactor. They are also helpful for optimizing operating parameters and reactor design.

\section{Conclusions}

Based on theoretical analysis and mathematical deduction, comprehensive correlations decribing the thickness of the water film and main operating parameters in TWR are proposed for the first time. These correlations can be used to calculate the thickness of the water film under knowing reactor sizes and some operating parameters. Also, the obtained quantitative correlation (Eq.(26)) shows that the thickness of the water film increases with the increase in the flow rate of transpiration water, but the flow rate cannot exceed the limitation value so as to guarantee the formation of good water film. Increasing the inlet temperature of transpiration water helps to improve the thickness of the water film. However, the high inlet temperature is also unfavorable for the low temperature protection for the transpiring wall. High reaction temperature can reduce the thickness of the water film, even results in its disappearance. As expected, there is a positive linear relation between transpiration water mass flow rate and the water film average temperature. 
In subsequent work, necessary in-depth experiments need conducted to verify and even correct these correlations.

\section{Acknowledgments}

This work is supported by the Projects from National Natural Science Foundation of China (No.21576219, No.21206132), and the Specialized Research Fund for the Doctoral Program of Higher Education (No.20120201120069), the Fundamental Research Funds for the Central Universities, the National Science Foundation for Post-doctoral Scientists of China (No. 2013M540748), the Special Financial Grant from the China Postdoctoral Science Foundation (NO. 2014T70922), and the Special Financial Grant from the Shaanxi Province Postdoctoral Science Foundation (No.126568).

\section{References}

[1] Z. Chen, G. Wang, Z.A. Mirza, S. Yang, Y. Xu, Study of transpiring fluid dynamics in supercritical water oxidation using a transparent reactor, The Journal of Supercritical Fluids 88 (2014) $117-125$.

[2] D. Xu, C. Huang, G. Lin, S. Wang, Y. Guo, Salt deposition problems in supercritical water oxidation, Chemical Engineering Journal 279 (2015) 1010-1022.

[3] P.A. Marrone, G.T. Hong, Corrosion control methods in supercritical water oxidation and gasification processes, Journal of Supercritical Fluids the 51(2) (2009) 83-103.

[4] M.D. Bermejo, M.J. Cocero, Destruction of an industrial wastewater by supercritical water oxidation in a transpiring wall reactor, Journal of Hazardous Materials 137(2) (2006) 965-971.

[5] K. Príkopský, B. Wellig, P.R. von Rohr, SCWO of salt containing artificial wastewater using a transpiring-wall reactor: Experimental results, The Journal of Supercritical Fluids 40(2) (2007) 
246-257.

[6] W. Gong, X. Duan, Degradation of landfill leachate using transpiring-wall supercritical water oxidation (SCWO) reactor, Waste Management 30(11) (2010) 2103-2107.

[7] D. Xu, S. Wang, C. Huang, X. Tang, Y. Guo, Transpiring wall reactor in supercritical water oxidation, Chemical Engineering Research and Design 92(11) (2014) 2626-2639.

[8] S.F. Rice, B.C. Wu, W.S. Winters, C.D. Robinson, Engineering Modeling of the Pine Bluff Arsenal Supercritical Water Oxidation Reactor, Subaru Proposal 17(1) (2013) 28-28.

[9] P.J. Crooker, K.S.A. And, Z. Fan, J. Prince, Operating Results from Supercritical Water Oxidation Plants, Industrial \& Engineering Chemistry Research 39(12) (2000) 4865-4870.

[10] D. Xu, S. Wang, X. Tang, Y. Gong, Y. Guo, Y. Wang, J. Zhang, Design of the first pilot scale plant of China for supercritical water oxidation of sewage sludge, Chemical Engineering Research and Design 90(2) (2012) 288-297.

[11] Z. Chen, G. Wang, F. Yin, H. Chen, Y. Xu, A new system design for supercritical water oxidation, Chemical Engineering Journal 269 (2015) 343-351.

[12] E. Fauvel, C. Joussot-Dubien, E. Pomier, P. Guichardon, G. Charbit, F. Charbit, S. Sarrade, Modeling Of A Porous Reactor For Supercritical Water Oxidation By A Residence Time Distribution Study, Industrial \& Engineering Chemistry Research 42(10) (2003) 2122-2130.

[13] E. Fauvel, C. Joussot-Dubien, P. Guichardon, G. Charbit, F. Charbit, S. Sarrade, A double-wall reactor for hydrothermal oxidation with supercritical water flow across the inner porous tube, Journal of Supercritical Fluids the 28(1) (2004) 47-56.

[14] W.J. Gong, F. Li, D.L. Xi, Supercritical Water Oxidation of Acrylic Acid Production Wastewater in Transpiring Wall Reactor, Environmental Engineering Science 26(1) (2009) 
$131-136$.

[15] D.C. Reda, Mixed convection in a liquid-saturated porous medium, Journal of Heat Transfer(United States) 110:1(1) (1988) 147-154.

[16] P. Jiang, B. Wang, D. Luo, Z. Ren, Fluid flow and convective heat transfer in a vertical porous annulus, Numerical Heat Transfer Applications 30(3) (1996) 305-320.

[17] M.D. Bermejo, F. Fdezpolanco, M.J. Cocero, Effect of the Transpiring Wall on the Behavior of a Supercritical Water Oxidation Reactor: Modeling and Experimental Results, Industrial \& Engineering Chemistry Research 45(10) (2006) 3438-3446.

[18] B. Wellig, K. Lieball, P.R.V. Rohr, Operating characteristics of a transpiring-wall SCWO reactor with a hydrothermal flame as internal heat source, The Journal of Supercritical Fluids 34(1) (2005) 35-50.

[19] M.D. Bermejo, F. Fdez-Polanco, M.J. Cocero, Experimental study of the operational parameters of a transpiring wall reactor for supercritical water oxidation, The Journal of Supercritical Fluids 39(1) (2006) 70-79.

[20] F. Zhang, S. Chen, C. Xu, G. Chen, J. Zhang, C. Ma, Experimental study on the effects of operating parameters on the performance of a transpiring-wall supercritical water oxidation reactor, Desalination 294 (2012) 60-66.

[21] C. Narayanan, C. Frouzakis, K. Boulouchos, K. Príkopský, B. Wellig, P. Rudolf von Rohr, Numerical modelling of a supercritical water oxidation reactor containing a hydrothermal flame, The Journal of Supercritical Fluids 46(2) (2008) 149-155.

[22] M.D. Bermejo, Á. Martín, J.P.S. Queiroz, I. Bielsa, V. Ríos, M.J. Cocero, Computational fluid dynamics simulation of a transpiring wall reactor for supercritical water oxidation, Chemical 
Engineering Journal 158(3) (2010) 431-440.

[23] S. Moussiere, A. Roubaud, O. Boutin, P. Guichardon, B. Fournel, C. Joussot-Dubien, 2D and 3D CFD modelling of a reactive turbulent flow in a double shell supercritical water oxidation reactor, The Journal of Supercritical Fluids 65 (2012) 25-31.

[24] F. Zhang, C. Ma, CFD simulation of a transpiring-wall SCWO reactor: Formation and optimization of the water film, Aiche Journal 62(1) (2015) 195-206.

[25] D.H. Xu, S.Z. Wang, Y.M. Gong, Y. Guo, X.Y. Tang, H.H. Ma, A novel concept reactor design for preventing salt deposition in supercritical water, Chemical Engineering Research and Design 88(11) (2010) 1515-1522.

[26] S.M. Yang, W.Q. Tao, Heat Transfer, fourth ed., Higher Education Press, Beijing, 2006, pp. 207-210. 